\title{
Solution structure of a dsDNA:LNA triplex
}

\author{
Jesper J. Sørensen, Jakob T. Nielsen and Michael Petersen* \\ Nucleic Acid Center, Department of Chemistry, University of Southern Denmark, 5230 Odense M, Denmark
}

Received July 18, 2004; Revised and Accepted October 27, 2004

\begin{abstract}
We have determined the NMR structure of an intramolecular dsDNA:LNA triplex, where the LNA strand is composed of alternating LNA and DNA nucleotides. The LNA oligonucleotide binds to the dsDNA duplex in the major groove by formation of Hoogsteen hydrogen bonds to the purine strand of the duplex. The structure of the dsDNA duplex is changed to accommodate the LNA strand, and it adopts a geometry intermediate between A- and B-type. There is a substantial propeller twist between base-paired nucleobases. This propeller twist and a concomitant large propeller twist between the purine and LNA strands allows the pyrimidines of the LNA strand to interact with the 5'flanking duplex pyrimidines. Altogether, the triplex has a regular global geometry as shown by a straight helix axis. This shows that even though the third strand is composed of alternating DNA and LNA monomers with different sugar puckers, it forms a seamless triplex. The thermostability of the triplex is increased by $19^{\circ} \mathrm{C}$ relative to the unmodified DNA triplex at acidic $\mathrm{pH}$. Using NMR spectroscopy, we show that the dsDNA:LNA triplex is stable at $\mathrm{pH} 8$, and that the triplex structure is identical to the structure determined at pH 5.1.
\end{abstract}

\section{INTRODUCTION}

Targeting duplex DNA with oligonucleotides offers an attractive method for artificial gene regulation through formation of a DNA triple helix (triplex) thereby modulating transcription of genes (the antigene strategy) or possibly inducing gene correction via recombinated repair (1-4). In a DNA triplex, the triplex-forming oligonucleotide (TFO) binds to a purinepyrimidine dsDNA duplex in the major groove through specific hydrogen bonds (5). The TFO can bind in both parallel and anti-parallel manners to the purine strand in the WatsonCrick duplex. In the parallel motif, a homopyrimidine TFO binds to the target duplex by Hoogsteen hydrogen bonding to form $\mathrm{T}: \mathrm{A} \bullet \mathrm{T}$ and $\mathrm{C}: \mathrm{G} \bullet \mathrm{C}^{+}$base triplets (Figure 1). Although appealing in theory, the antigene strategy is severely hampered by two factors owing to the very nature of triplexes: (i) In the Hoogsteen hydrogen bond arrangement, only purinepyrimidine tract dsDNA can be targeted and (ii) the cytosine bases in the TFO must be protonated at $\mathrm{N} 3$ to form $\mathrm{C}: \mathrm{G} \cdot \mathrm{C}^{+}$ triplets, thus requiring un-physiological acidic conditions. a $\operatorname{man}_{\alpha}$

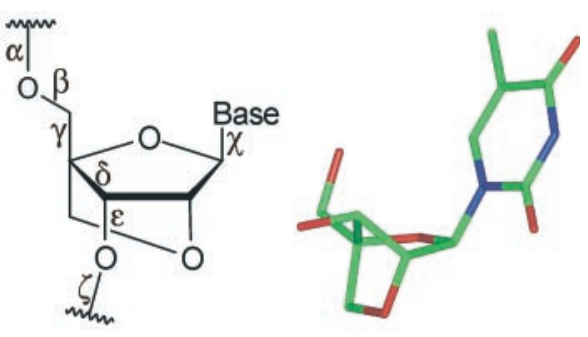

b

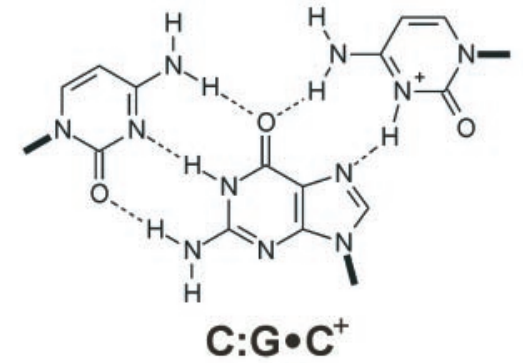

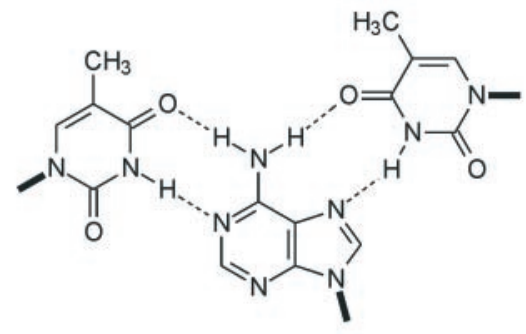

$\mathrm{T}: \mathrm{A} \bullet \mathrm{T}$

Figure 1. Locked nucleic acid and the base triplets in triple helical nucleic acids (a) The chemical structure of LNA, the nucleic acid backbone angles are indicated (left), and stick plot showing the $\mathrm{C} 3^{\prime}$-endo sugar pucker (right). (b) The $\mathrm{T}: \mathrm{A} \bullet \mathrm{T}$ and $\mathrm{C}: \mathrm{G} \bullet \mathrm{C}^{+}$base triplets.

Problem (i) is addressed by synthesis of unnatural nucleobases that target A:T and G:C Watson-Crick base pairs(6). Problem (ii) is somewhat alleviated by use of high-affinity nucleic acid analogues, of which LNA (locked nucleic acid) is prominent (Figure 1) (7).

LNA contains a $2^{\prime}-O, 4^{\prime}-C$ methylene bridge which locks the sugar in a perfect $\mathrm{C} 3^{\prime}$-endo ( $N$-type, RNA-like) conformation (8). LNA stabilizes DNA and RNA duplexes by up to $10^{\circ} \mathrm{C}$ per incorporation, and appears as a most promising nucleic acid analogue with applications in diagnostics and oligonucleotidebased therapeutics $(9,10)$. The high-affinity hybridization properties of LNA encompass dsDNA recognition as well, and allow formation of dsDNA:LNA triplexes at physiological

*To whom correspondence should be addressed. Tel: +45 6550 2530; Fax: +45 6615 8780; Email: mip@chem.sdu.dk 
a

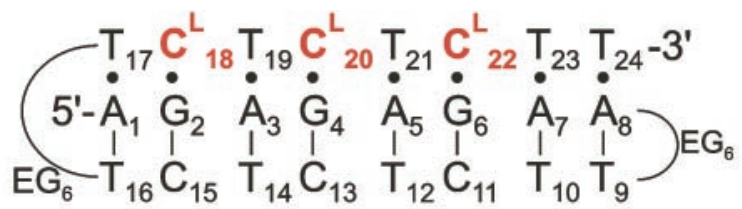

b

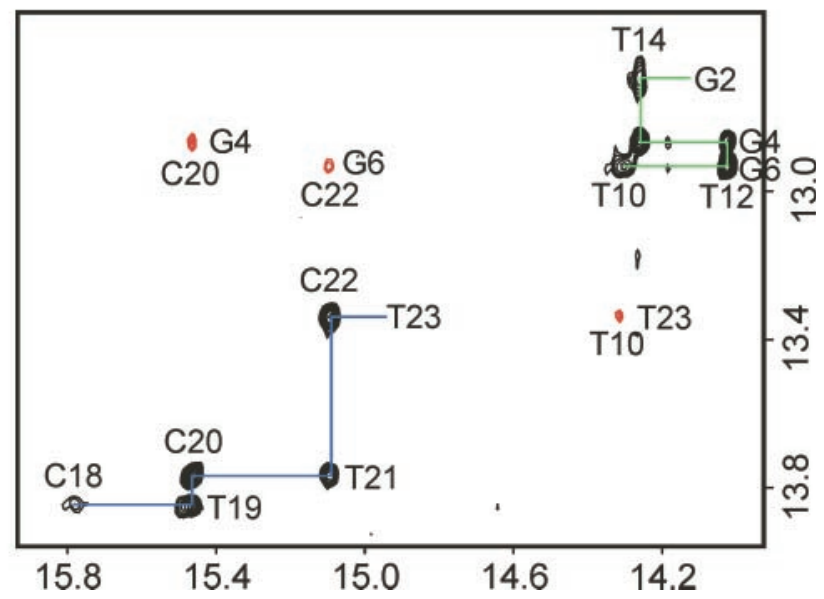

Figure 2. NMR structure determination of the dsDNA:LNA triplex. (a) Sequence of the triplex. (b) $800 \mathrm{MHz}{ }^{1} \mathrm{H},{ }^{1} \mathrm{H}$ NOESY spectrum acquired in $\mathrm{H}_{2} \mathrm{O}\left(\mathrm{pH} 5.1,25^{\circ} \mathrm{C}\right)$. The imino-imino region is shown Sequential assignments in the Watson-Crick duplex (green) and LNA TFO strand (blue) are shown together with cross-peaks between the TFO and the duplex part of the triplex (red).

$\mathrm{pH}$. As such, incorporation of five or seven LNA monomers in a 15mer DNA TFO raised the melting temperature of the triplex to duplex transformation from 33 to $\sim 60^{\circ} \mathrm{C}$ at $\mathrm{pH} 6.8$ (11). These optimum results were obtained with TFOs composed of alternating DNA and LNA monomers, while intriguingly fully modified LNA TFOs do not form triplexes at $\mathrm{pH}$ values above $\sim 6$ (12). From analysis of circular dichroism (CD) spectra, it was inferred that dsDNA:LNA triplexes have a more A-like structure than triplexes with native DNA TFOs, whilst thermodynamic data suggested that hydrogen bonding and base stacking is significantly altered when hybridizing with an LNA TFO rather than a DNA TFO (11).

In an attempt to rationalize LNA's triplex-forming properties and to provide structural information at the atomic level, we have determined the NMR structure of an intramolecular dsDNA:LNA triplex, where the LNA TFO is composed of alternating LNA and DNA nucleotides (Figure 2).

\section{MATERIALS AND METHODS}

\section{Experimental details}

The modified oligonucleotide was synthesized as described elsewhere (13). The oligonucleotide was purified by siteexclusion on a Sephadex G15 column. The sample concentration was $1.8 \mathrm{mM}$ triplex and $100 \mathrm{mM} \mathrm{NaCl}$ in $0.5 \mathrm{ml} \mathrm{D}_{2} \mathrm{O}$ or 9:1 $\mathrm{H}_{2} \mathrm{O} / \mathrm{D}_{2} \mathrm{O}(\mathrm{pH}=5.1)$. NMR spectra were recorded at Varian Inova 500 and Varian Inova 800 spectrometers. For assignment purposes, standard 2D NOESY (in $\mathrm{D}_{2} \mathrm{O}$ and $\mathrm{H}_{2} \mathrm{O}$ ),
DQF-COSY and 3D TOCSY-NOESY experiments were acquired.

For the structure determination, NOESY spectra in $\mathrm{D}_{2} \mathrm{O}$ were acquired at $35^{\circ} \mathrm{C}$ at $800 \mathrm{MHz}$ with mixing times of $50,110,180$ and $250 \mathrm{~ms}$ and a NOESY spectrum in $\mathrm{H}_{2} \mathrm{O}$ was acquired at $25^{\circ} \mathrm{C}$ at $800 \mathrm{MHz}$ with a mixing time of $200 \mathrm{~ms}$. The spectra in $\mathrm{D}_{2} \mathrm{O}$ were acquired using 2048 complex points in $t_{2}$ and a spectral width of $8000 \mathrm{~Hz}$. A total of $400 \mathrm{t}_{1^{-}}$ experiments, each with 32 scans and a dwell-time of $4.0 \mathrm{~s}$ between scans, were recorded using the States phase cycling scheme. The residual signal from HOD was removed by lowpower presaturation. The spectrum in $\mathrm{H}_{2} \mathrm{O}$ was acquired using the WATERGATE NOESY pulse sequence using $2048 \mathrm{com}-$ plex points in $t_{2}$ and a spectral width of $19230 \mathrm{~Hz}$. A total of $712 t_{1}$-experiments, each with 32 scans and a dwell time of $3.1 \mathrm{~s}$ between scans, were recorded using the States phase cycling scheme. In addition to the NOESY spectra, an inversion recovery experiment was recorded at $800 \mathrm{MHz}$ to extract $\mathrm{T}_{1}$ relaxation rates.

To gauge sugar conformations of the deoxyribo nucleotides, a DQF-COSY spectrum was acquired at $500 \mathrm{MHz}$ at $35^{\circ} \mathrm{C}$ using a pulse sequence in which the first pulse was replaced with an E-BURP type selective pulse (14) in order to enhance the digital resolution in F1. This spectrum was acquired with spectral widths of $5000 \mathrm{~Hz}$ in F2 and $1200 \mathrm{~Hz}$ in F1, respectively, and a total of $580 \mathrm{t}_{1}$-experiments, each with $80 \mathrm{scans}$, with 2048 complex points in $\mathrm{t}_{2}$. 1D spectra in $\mathrm{H}_{2} \mathrm{O}$ were recorded at $25^{\circ} \mathrm{C}$ with various $\mathrm{pH}$ values in the range 4.98.0. The acquired data were processed using FELIX (ver. 98; Accelrys Inc.). For the structure determination, the spectra recorded in $\mathrm{D}_{2} \mathrm{O}$ were linear predicted to 600 points in $\mathrm{F}_{1}$, and the spectrum recorded in $\mathrm{H}_{2} \mathrm{O}$ was linear predicted to 1000 points in $\mathrm{F}_{1}$. All spectra were baseline corrected by the FLATT procedure in $\mathrm{F}_{1}(15)$, and by the automatic baseline correction procedure as implemented in FELIX in $\mathrm{F}_{2}$.

\section{Distance restraints}

A total of 425 distance restraints were obtained from 2D NOE cross-peak intensities using the method of Wijmenga and van Buuren. (16) in this variation of the isolated spin pair approximation, spin diffusion is accounted for in an average manner. NOESY cross-peak intensities from the four spectra recorded in $\mathrm{D}_{2} \mathrm{O}$ were corrected for saturation effects using $\mathrm{T}_{1}$ relaxation times obtained from an inversion recovery experiment and were subsequently transformed to distance restraints by calibrating against known distances. The final upper and lower distance bounds used in the structure determination were determined from the standard deviations calculated by performing the procedure 100 times with slightly perturbed NOESY volumes. This resulted in the bounds being on average $\pm 12 \%$ of the average distance obtained from the 100 calculations. A total of 287 restraints were derived from the NOESY spectra recorded in $\mathrm{D}_{2} \mathrm{O}$. From the NOESY spectrum recorded in $\mathrm{H}_{2} \mathrm{O}$, a further 138 restraints including exchangeable protons were derived by a procedure as described above, the upper and lower distance bounds were set to the distances calculated $\pm 20 \%$. The distribution of NOE restraints for the dsDNA:LNA triplex is included in Table 1. The average width of the distance restraints was $1.17 \AA$, and the average restraint length $3.85 \AA$. All distance restraints were incorporated into 
Table 1. Structural statistics for the dsDNA:LNA triplex

\begin{tabular}{lc}
\hline Structural restraints & \\
NOE-derived distance restraints & 425 \\
Intra-nucleotide & 183 \\
Inter-nucleotide, intra-strand & 147 \\
Inter-strand & 95 \\
$1-2^{\mathrm{a}}$ & 21 \\
$1-3^{\mathrm{a}}$ & 66 \\
$2-3^{\mathrm{a}}$ & 8 \\
Hydrogen bond restraints & 35 \\
Violations of restraints & \\
Average number of violations $>0.2 \AA$ & 1.5 \\
Maximum violation $(\AA)$ & 0.31 \\
Atomic rmsd $(\AA)^{\mathrm{b}}$ & \\
All nucleotides & 0.91 \\
Internal nucleotides & 0.77 \\
\hline
\end{tabular}

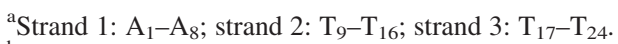

${ }^{\mathrm{b}}$ Average pair-wise all atomic rmsd for the ensemble of 20 structures calculated.

the AMBER potential energy by a standard flat-well pseudo potential.

Watson-Crick and Hoogsteen base-pairing was inferred from the NOESY spectrum acquired in $\mathrm{H}_{2} \mathrm{O}$ and consequently 35 hydrogen bond distance restraints were included in the calculations. Target values for these restraints were taken from crystallographic data (17).

\section{Structure calculations}

All calculations were performed with the AMBER5 suite of programs (18). A simulated annealing (SA) protocol was utilized to obtain the structure of the triplex. Two different starting structures were employed in the calculations, the isosequential dsDNA:RNA and dsDNA:DNA structures determined by Feigon and co-workers (pdb accession codes: $1 \mathrm{r} 3 \mathrm{x}$ and $1 \mathrm{~d} 3 \mathrm{x})$. The appropriate nucleotides were modified to LNA nucleotides, and atomic charges for the modified nucleotides were calculated using the RESP procedure (19).

Initially, the starting structure was restrained energy minimized before being subjected to 80 ps of molecular dynamics in time-steps of $1 \mathrm{fs}: 10 \mathrm{ps}$ at $1500 \mathrm{~K}$ followed by cooling to $300 \mathrm{~K}$ over $70 \mathrm{ps}$. Finally, a further restrained energy minimization was carried out. In the SA scheme, the force constant for distance restraints was $50 \mathrm{kcal} /\left(\mathrm{mol} \AA^{2}\right)$. A distance-dependent dielectric constant, $\varepsilon=4 r$, was used and the non-bonded cut-off was $30 \AA$.

\section{Structure analysis}

Structural statistics were derived for the 20 calculated structures. Helix parameters were calculated with Curves 5.3 $(20,21)$. Root mean square deviations (rmsds) between observed and back-calculated proton chemical shifts in the structural ensemble were calculated with Nuchemics $(22,23)$.

\section{Thermal stability studies}

The thermal stability of the triplex was determined spectrophotometrically at $\mathrm{pH}$ values of 4.6, 5.9, 6.8 and 8.4 with a spectrophotometer equipped with a thermoregulated Peltier element. The samples were prepared by dissolving $\sim 3 \mu \mathrm{M}$ of the oligonucleotide in $1 \mathrm{ml}$ containing $100 \mathrm{mM} \mathrm{NaCl}$. The $\mathrm{pH}$ value was adjusted using $\mathrm{NaOH}$ and $\mathrm{HCl}$. The absorbance at $260 \mathrm{~nm}$ was monitored while the temperature was raised linearly from 10 to $93^{\circ} \mathrm{C}\left(1^{\circ} \mathrm{C} / \mathrm{min}\right)$. The melting temperatures ( $T_{\mathrm{m}}$ value) were obtained as the maxima of the first derivative of the melting curves.

\section{RESULTS \\ NMR analysis}

The sequence of the intramolecular dsDNA:LNA triplex is shown in Figure 2. We chose this sequence as the structures of the corresponding dsDNA:RNA and dsDNA:DNA triplexes have been determined previously by Feigon and co-workers $(24,25)$. The TFO is composed of alternating DNA and LNA nucleotides as this appears to be the optimum design for LNA TFOs. For synthetic reasons, the LNA cytosine nucleotides are 5 -methylcytosines. The insertion of a 5-methyl group is known to stabilize triplex formation by $1-2^{\circ} \mathrm{C}$ per methyl group $(12,26)$. This stabilization presumably stems from increased stacking interactions (27).

The dsDNA:LNA triplex was assigned and analysed using standard 2D and 3D NMR experiments, including NOESY, COSY and TOCSY-NOESY, at $35^{\circ} \mathrm{C}\left(25^{\circ} \mathrm{C}\right.$ for experiments examining exchangeable protons) and $\mathrm{pH}$ 5.1. The NOESY spectra recorded in $\mathrm{H}_{2} \mathrm{O}$ display the characteristic connectivities showing Watson-Crick base-pairing between the duplex part of the molecule. Triplex formation with the TFO was confirmed by observation of cross-peaks between (i) the H8 and the imino protons of Hoogsteen base-paired residues, (ii) the amino protons of the two cytosine residues in each base triplet and (iii) the $\mathrm{H} 8$ of the purine residues and the $\mathrm{H} 1^{\prime}$ of the $3^{\prime}$-flanking residue in the TFO strand. The formation of the eight possible triplets was confirmed. An excerpt of the $200 \mathrm{~ms}$ NOESY spectrum recorded in $\mathrm{H}_{2} \mathrm{O}$ is shown in Figure 2. The three cytosines in the TFO strand are protonated at N3 as shown by the lines from $\mathrm{H} 3$ in the low-field part of the spectrum in $\mathrm{H}_{2} \mathrm{O}$ (see Figure 2), and by the distinctly downfield shifted amino proton resonances of the protonated cytosines in the TFO strand. We thus conclude that the LNA TFO binds to the Watson-Crick duplex by Hoogsteen base-pairing. The ethylene-glycol linker was not specifically assigned and was not included in the structure calculations.

\section{The dsDNA:LNA triplex structure}

The structure of the dsDNA:LNA triplex was determined using 425 NOE restraints and 35 restraints to mimic WatsonCrick and Hoogsteen base-pairing (Table 1). No torsion angle restraints were included for the sugar-phosphate backbone. Rather information on the sugar-phosphate backbone was used to validate the structure generated with the NOE restraints. We chose this approach as we found that the NOE restraints themselves are defining the structure with good precision. The final structural ensemble of 20 structures has a rmsd of $0.91 \AA$ for all triplets, and $0.77 \AA$ for the six internal triplets (Table 2). This shows that the structure overall is well defined by the NOE restraints employed. The nucleobases are better defined in the structure than the sugarphosphate backbone (see Figure 3). We performed test calculations without hydrogen bond restraints, and the ensemble thus determined was identical to the structural ensemble, although the rmsd was higher. In addition, we performed 
Table 2. Structural parameters for the dsDNA:LNA structure ${ }^{a}$

\begin{tabular}{lllll}
\hline Structure $^{\mathrm{b}}$ & $\begin{array}{l}E_{\mathrm{AMBER}} \\
(\mathrm{kcal} / \mathrm{mol})\end{array}$ & $\begin{array}{l}E_{\mathrm{NOE}} \\
(\mathrm{kcal} / \mathrm{mol})\end{array}$ & $\begin{array}{l}\Delta d_{\mathrm{av}} \\
(\AA)\end{array}$ & $\begin{array}{l}\text { rmsd vs NMR } \\
(\AA)\end{array}$ \\
\hline dsDNA:DNA & -40.1 & 1721.8 & 0.127 & $1.48(0.10)$ \\
dsDNA:RNA & -19.9 & 2356.9 & 0.168 & $1.78(0.09)$ \\
NMR & $-9.9(7.8)$ & $22.9(2.0)$ & $0.011(0.0005)$ & \\
\hline
\end{tabular}

${ }^{\mathrm{a}}$ Force field $\left(E_{\mathrm{AMBER}}\right)$ and restraint $\left(E_{\mathrm{NOE}}\right)$ energies, average restraint violations $\left(\Delta d_{\mathrm{av}}\right)$ and pair wise atomic rmsds for the starting structures and the NMR structure. Standard deviations are shown in brackets.

${ }^{b}$ The starting structures are the isosequential triplexes, pdb codes: $1 \mathrm{~d} 3 \mathrm{x}$ and $1 \mathrm{r} 3 \mathrm{x}$, respectively, modified with LNA nucleotides and energy minimized. twenty sets of calculations in each of which we randomly deleted $10 \%$ of the NOE restraints. The structures generated in this manner all converge well and yield structures identical to the structure obtained with the maximal NOE restraint set. Thus, the triplex structure is not strongly dependent on a few of the NOE restraints.

Chemical shifts are sensitive parameters to probe local geometry, and we back-calculated the chemical shifts for the duplex part of the dsDNA:LNA triplex. The rmsd between observed and back-calculated chemical shifts for aromatic and $\mathrm{H}^{\prime}$ protons is $0.21 \mathrm{ppm}$ for non-terminal nucleotides. a

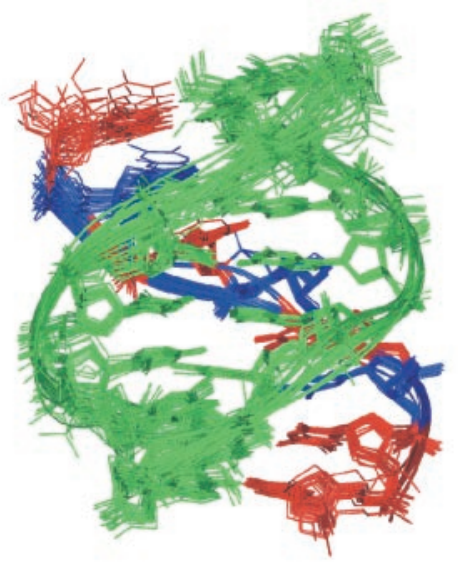

C

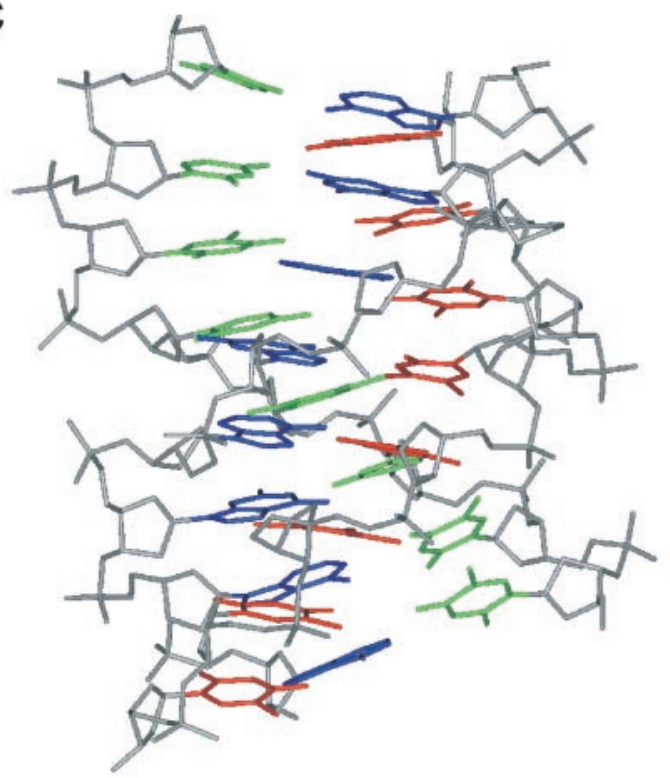

b
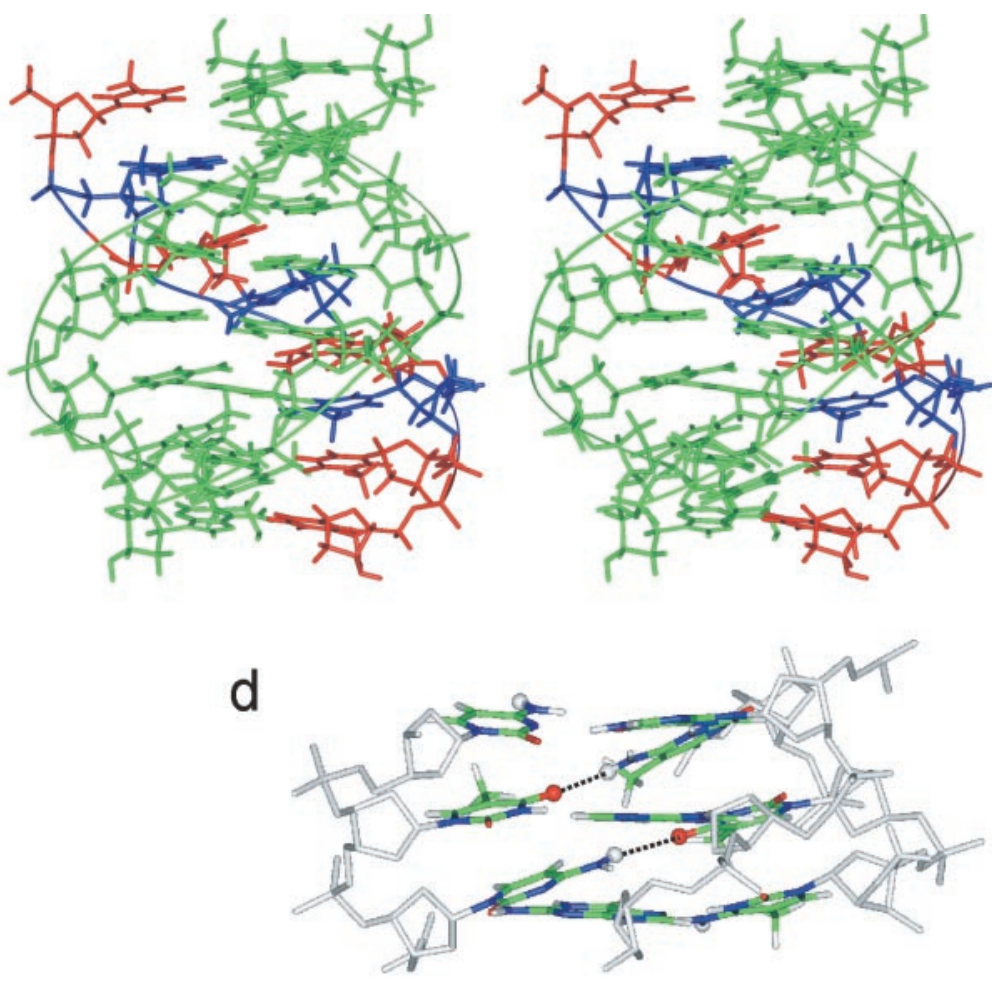

e

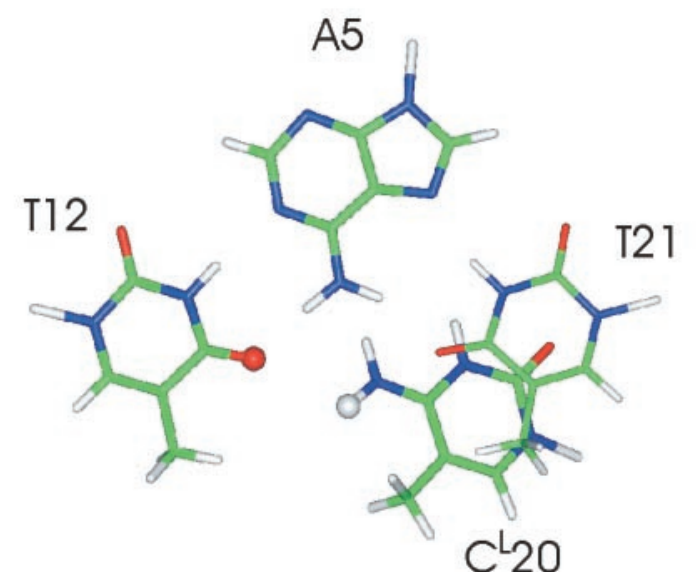

Figure 3. Solution structure of the dsDNA:LNA triplex. (a) Superposition of the 20 structures calculated. The Watson-Crick duplex is green and the TFO is red (DNA) and blue (LNA). The rmsd of the 20 structures is $0.91 \AA$. (b) Stereo view of a representative structure of the triplex. (c) View showing the spiral-like arrangement of the nucleobases. The purine bases are blue, the Watson-Crick pyrimidines green and the TFO pyrimidines red. For clarity, only heavy atoms are shown. (d) Close up of the pyrimidine-pyrimidine hydrogen bonding, the $\mathrm{C}_{13}-\mathrm{G}_{4}-\mathrm{C}_{20}^{\mathrm{L}}, \mathrm{T}_{12}-\mathrm{A}_{5}-\mathrm{T}_{21}$ and $\mathrm{C}_{11}-\mathrm{G}_{6}-\mathrm{C}^{\mathrm{L}}{ }_{22}$ triplets are shown. The $\mathrm{O} 4$ atoms of the thymines are red spheres and the $\mathrm{H} 4$ atoms of cytosines white spheres. (e) View along the helix axis. 
We observed large deviations ( $>0.5$ p.p.m.) for two protons, $\mathrm{A}_{3} \mathrm{H} 2$ and $\mathrm{A}_{5} \mathrm{H} 8$. Excluding these chemical shifts reduced the rmsd to 0.16 p.p.m.

The Watson-Crick part of the triplex adopts an overall geometry intermediate between A- and B-helix types as indicated by a number of helical parameters, e.g. $X$ displacement: $-3.0 \AA$, inclination: $9.9^{\circ}$, rise: $3.0 \AA$ and propeller twist: $-21^{\circ}$. The average twist between the six internal nucleobases is $34^{\circ}$, whilst the two terminal base-pair steps are unwound at $27^{\circ}$ and $30^{\circ}$. Overall, the distribution of helix parameters along the duplex is uniform. The average minor groove width of the duplex is $6.1 \AA$. The sugar conformations in the Watson-Crick part of the molecule is in the $S$-type domain with pseudorotation values ranging from $99^{\circ}$ to $192^{\circ}$, except for $\mathrm{C}_{15}\left(P=67^{\circ}\right)$. These sugar conformations are corroborated by inspection of $\mathrm{H} 1^{\prime}-\mathrm{H} 2^{\prime} / \mathrm{H} 2^{\prime \prime}$ cross-peaks in COSY spectra, as these cross-peaks show the distinct fine structure of furanoses with predominantly (>80\%) $S$-type sugar puckers, except for $\mathrm{C}_{15}$ 's cross-peaks, which show a higher population of $\mathrm{N}$-type conformation.

The TFO LNA strand binds to the Watson-Crick duplex in the major groove, and we observe formation of eight triplets as indicated by analysis of the NOESY data. The TFO strand divides the major groove into two unequal grooves with widths approximately in the ratio $1: 2$. The $2^{\prime}-O, 4^{\prime}-C$ bridges of the LNA nucleotides are located at the brim of the narrow groove with the $2^{\prime}$-oxygen pointing into this groove. The puckering amplitude of the LNA sugar is large $\left(\sim 60^{\circ}\right)$. As a consequence, the $3^{\prime}$-carbons are protruding into the larger of the grooves, and the $3^{\prime}$-hydrogens and the methyl groups of the $3^{\prime}$-flanking thymidines are in close proximity. The sugar puckers in the TFO strand are mixed with the LNA nucleotides $\left(\mathrm{C}_{18}^{\mathrm{L}}, \mathrm{C}^{\mathrm{L}}{ }_{20}, \mathrm{C}^{\mathrm{L}}{ }_{22}\right), \mathrm{T}_{23}$ and $\mathrm{T}_{24}$ having $N$-type conformations and the remaining thymidines $\left(\mathrm{T}_{17}, \mathrm{~T}_{19}\right.$ and $\left.\mathrm{T}_{21}\right) S$-type puckers. As for the duplex part of the molecule, the sugar puckers of the TFO are in agreement with COSY cross-peaks, although $\mathrm{T}_{19}$ and $\mathrm{T}_{21}$ show peaks characteristic of $N / S$ equilibriums with a substantial $N$-type population $(\sim 30 \%)$.

The torsion angles defining the sugar-phosphate backbone of the three strands generally fall in the expected range for a right-handed nucleic acid, i.e. $\alpha$ to $\gamma$ : gauche-, trans, gauche+, gauche+, trans and gauche-. Conspicuous exceptions are the $\beta$ and $\gamma$ angles of $A_{3}$ and $A_{5}$, with the $\beta$ angles adopting multiple rotamers and the $\gamma$ angles adopting exclusively trans conformations. In addition, the glycosidic angle, $\chi$, has rather low values for $\mathrm{A}_{3}$ and $\mathrm{A}_{5},-154^{\circ}$ and $-157^{\circ}$, as compared to an average of $-118^{\circ}$ for the remaining nucleotides in the Watson-Crick part of the molecule. This alteration in the sugar-phosphate backbone geometry is owing to the phosphates $\mathrm{G}_{2} \mathrm{pA}_{3}$ and $\mathrm{G}_{4} \mathrm{pA}_{5}$ being pushed towards the TFO strand while the nucleobases $\mathrm{A}_{3}$ and $\mathrm{A}_{5}$ remain in a continuous stack with the other purines. A similar arrangement was found by Asensio et al. in a $5^{\prime}-$ GAGAG- $3^{\prime}$ context (pdb entry: $1 \mathrm{bwg}$ ), and as such appears sequence specific rather than owing to the introduction of LNA monomers in the TFO (28). We observe distinct downfield shifts of the $\mathrm{H}^{\prime}$ resonances in $\mathrm{A}_{3}$ and $\mathrm{A}_{5}$ in accordance with the observations made by Asensio et al. The change in backbone geometry allows the negatively charged phosphate group to move closer to the protonated nucleobases of the cytidines in the TFO strand. Besides this, the two phosphate groups in question are close to the $\mathrm{O} 2^{\prime}$ oxygens of the LNA monomers ( $\mathrm{P}-\mathrm{O}$ distance $\sim 4-5 \AA$ ). This distance indicates that a water-mediated interaction is possible although there is no experimental evidence for this.

Altogether, the triplex is regular in its global geometry as shown by a straight helix axis and uniform helix parameters along the triplex. This shows that even though the TFO is composed of alternating DNA and LNA monomers with different sugar puckers, it forms a seamless triplex.

The propeller twist between nucleobases in the WatsonCrick base pairs is rather large (average $-21^{\circ}$ ); in addition, a comparable large propeller twist is found in the Hoogsteen base pairs between the pyrimidines in the TFO and the purines of the duplex (average $-14^{\circ}$ ). A consequence of this is that the pyrimidine bases of the TFO strand are close to the bases of the $5^{\prime}$-flanking pyrimidines of the duplex. In fact, the amino groups and the $\mathrm{O} 4$ oxygens of the TFO cytosines and thymines, respectively, are able to partake in bifurcated hydrogen bonds with both the Hoogsteen-paired purine and the $5^{\prime}$-flanking pyrimidine of the Watson-Crick duplex (see Figure 3). This creates a spiral-like hydrogen bonding pattern through the triplex. The hydrogen bonds between the pyrimidines are not as strong as either the Watson-Crick or Hoogsteen hydrogen bonds, as gauged by their geometries (Table 3). It is observed that the best bonding geometries are found when the donor is a TFO LNA-cytidine. The bifurcated hydrogen bonds are somewhat reminiscent of what is observed in A-tract DNA (29).

For four of the $\mathrm{C}^{*} \mathrm{~T}$ pyrimidine-pyrimidine base pairs, the $\mathrm{Cl}^{\prime}$ and $\mathrm{O} 4$ atoms of the thymidines, and the $\mathrm{N} 4$ and $\mathrm{Cl}^{\prime}$ atoms of the cytidines are placed in a linear geometry (see Figure 3). This could be the average geometry of a slightly wobbling cytosine base presenting either of the amino protons for hydrogen bonding with the $\mathrm{O} 4$ atom of thymine.

\section{Triplex formation at neutral $\mathrm{pH}$}

To evaluate the stability of the intramolecular dsDNA:LNA triplex, we recorded 1D ${ }^{1} \mathrm{H}-\mathrm{NMR}$ spectra in $\mathrm{H}_{2} \mathrm{O}$ and measured melting temperatures, $T_{m}$ values, as a function of $\mathrm{pH}$. As seen in Figure 4, the signals from the $\mathrm{H} 3$ protons of the protonated cytidines remain intact at all $\mathrm{pH}$ values up to 8.0. This indicates that the cytosines remain protonated at $\mathrm{pH}$ 8.0. The line of the $\mathrm{H} 3$ proton of $\mathrm{C}_{18}$ is broad at all $\mathrm{pH}$ values, thus indicating faster exchange with the solvent than for $\mathrm{C}_{20}$ and $\mathrm{C}_{22}$. This is possibly caused by the ethylene-glycol linker being slightly too short to bridge the distance between $T_{16}$ and $\mathrm{T}_{17}$, thus causing some disruptions to the terminal triplet (30). The apparent $\mathrm{p} K_{\mathrm{a}}$ in unmodified DNA triplexes depend on the number and position of $\mathrm{C}: \mathrm{G} \cdot \mathrm{C}^{+}$triplets. Terminal $\mathrm{C}: \mathrm{G} \cdot \mathrm{C}^{+}$triplets and $\mathrm{C}: \mathrm{G} \cdot \mathrm{C}^{+}$triplets in mixed sequences

Table 3. Statistics of pyrimidine-pyrimidine hydrogen bonds

\begin{tabular}{lllll}
\hline Donor & Acceptor & Distance $(\AA)$ & Angle $\left(^{\circ}\right)^{\mathrm{a}}$ & Occupancy $(\%)^{\mathrm{b}}$ \\
\hline $\mathrm{C}_{11}: \mathrm{N} 4$ & $\mathrm{~T}_{21}: \mathrm{O} 4$ & 3.17 & 56 & 30 \\
$\mathrm{C}_{13}: \mathrm{N} 4$ & $\mathrm{~T}_{19}: \mathrm{O} 4$ & 3.48 & 54 & 50 \\
$\mathrm{C}_{15}: \mathrm{N} 4$ & $\mathrm{~T}_{17}: \mathrm{O} 4$ & 3.64 & 56 & 20 \\
$\mathrm{C}^{\mathrm{L}}{ }_{18}: \mathrm{N} 4$ & $\mathrm{~T}_{14}: \mathrm{O} 4$ & 3.15 & 47 & 95 \\
$\mathrm{C}^{\mathrm{L}}{ }_{20}: \mathrm{N} 4$ & $\mathrm{~T}_{12}: \mathrm{O} 4$ & 3.15 & 47 & 100 \\
$\mathrm{C}^{\mathrm{L}}{ }_{22}: \mathrm{N} 4$ & $\mathrm{~T}_{10}: \mathrm{O} 4$ & 3.71 & 43 & 100 \\
\hline
\end{tabular}

${ }^{\mathrm{a} D}$ Deviation from linearity.

${ }^{\mathrm{b}}$ Percentage of the calculated structures in which the hydrogen bond occurs (cut-off values are distance $<4 \AA$ and angle $<60^{\circ}$ ). 


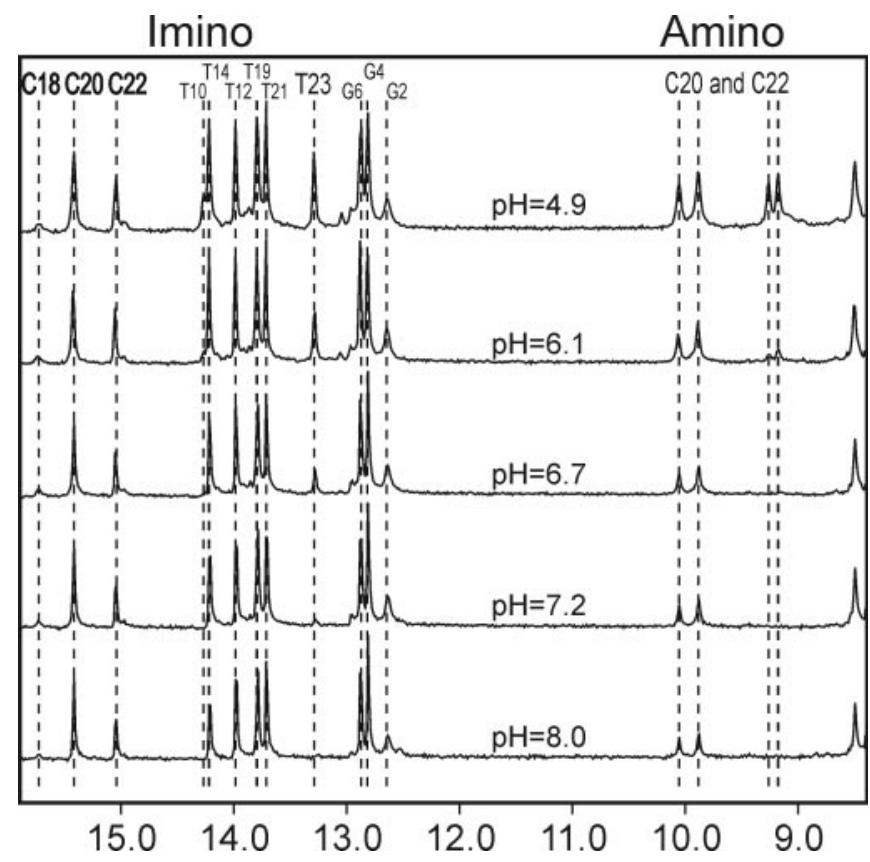

Figure 4. Triplex formation at neutral pH. $1 \mathrm{D}{ }^{1} \mathrm{H}$ NMR spectra of the imino and the down field amino proton regions at various $\mathrm{pH}$ values.

have apparent $\mathrm{p} K_{\mathrm{a}}$ values of $\sim 7-8,(31,32)$ while internal $\mathrm{C}: \mathrm{G} \bullet \mathrm{C}^{+}$triplets in contiguous $\mathrm{T}: \mathrm{A} \bullet \mathrm{T}$ stretches have higher apparent $\mathrm{p} K_{\mathrm{a}}$ values (33). We did not determine the apparent $\mathrm{p} K_{\mathrm{a}}$ values of the cytosines in the dsDNA:LNA triplex, but our results show that the apparent $\mathrm{p} K_{\mathrm{a}}$ is raised substantially from unmodified DNA triplexes.

To assess whether there occurred structural changes in the triplex, we recorded a NOESY spectrum in $\mathrm{H}_{2} \mathrm{O}$ at $\mathrm{pH} 8.0$ $\left(25^{\circ} \mathrm{C}\right)$. In this spectrum, we observe a cross-peak pattern analogous to that in the spectra at $\mathrm{pH} 5.1$. We do observe some cross-peaks that could not be assigned to the triplex; it is most likely that these cross-peaks stem from the WatsonCrick duplex with the TFO dissociated. From the volumes of these cross-peaks, we estimate that at $\mathrm{pH} 8.0$ at least $80 \%$ of the sample is still found as triplex. From the similarity of the NOESY cross-peak patterns, we conclude that the structure of the triplex at $\mathrm{pH} 8.0$ is unchanged from the structure determined at $\mathrm{pH} 5.1$.

The melting temperatures measured accentuate the stability of the LNA-modified triplex. In the interval from $\mathrm{pH} 4.6$ to 8.4, the melting temperature decreases from 88 to $58^{\circ} \mathrm{C}$. This can be compared with melting temperatures of $\sim 69^{\circ} \mathrm{C}$ (at $\mathrm{pH}$ 5.2) for the dsDNA:DNA and dsDNA:RNA versions of the triplex measured under conditions with slightly higher ionic strength $(24,25)$. At $\mathrm{pH}<7$, only one melting transition is observed for the triplex, whilst at $\mathrm{pH} 8.4$ the melting transition is broad, thus indicating that the duplex to triplex and the duplex to coil transitions are separating.

\section{DISCUSSION}

Our structure of the dsDNA:LNA triplex reveals a spiral-like hydrogen bonding and stacking motif. Analysis of the triplex structures deposited with the Protein Data Bank reveals one structure (entry 1bwg; mentioned above) with an arrangement of nucleobases somewhat resemblant of this dsDNA:LNA triplex (28). The 1 bwg structure contains a $5^{\prime}$-CTCTCT- $3^{\prime}$ TFO, i.e. identical to part of our LNA TFO. The spiral-like pattern is less pronounced in the $1 \mathrm{bwg}$ structure and the putative pyrimidine-pyrimidine hydrogen bonds have geometries less optimal for interactions as gauged from the stagger between the cytosine and thymine nucleobases.

In the structure of the unmodified DNA triplex of identical composition (pdb: 1d3x), all triplets are almost planar (24). However, as explicit base planarity restraints were included in the structure calculations of the unmodified triplex, it is difficult to make a direct comparison. We did attempt to put planarity restraints on base triplets in the dsDNA:LNA triplex, but with force constants of $15 \mathrm{kcal} / \mathrm{mol} \mathrm{rad}^{2}$, we could only force the terminal triplets planar. These calculations validate the stacking and hydrogen bond motif in the dsDNA:LNA triplex as they show that the motif is well determined by the experimental NOEs. The structure cannot reveal whether the pyrimidine-pyrimidine interactions are the cause or a consequence of the large propeller twists between the strands. However, as these interactions probably are rather weak, it appears likely that the structural rearrangement is mainly caused by the accommodation of the LNA TFO.

In the context of LNA:DNA duplexes, we have previously shown that deoxyriboses located between two LNA nucleotides are steered into pure $N$-type puckers (34). This is not the case for the thymidines between the LNA cytidines in the TFO which shows that LNA nucleotides impose different constraints on neighbouring nucleotides in duplexes and triplexes. Furthermore, it demonstrates that in the context of a dsDNA:LNA triplex, it is not favourable to have a TFO strand where the sugars are entirely in $N$-type conformations. This could explain why it is unfavourable to have a fully LNAmodified TFO. Most intriguingly, a close analogue of LNA, ENA $\left(2^{\prime}-O, 4^{\prime}-C\right.$-ethylene-bridged nucleic acid) with the $\mathrm{O} 2^{\prime}$ and $\mathrm{C}^{\prime}$ atoms bridged by an ethylene linker, forms triplexes with fully modified TFOs at neutral $\mathrm{pH}$ (35). A nucleoside $\mathrm{X}$-ray structure shows that ENA adopts a C3'-endo sugar pucker as LNA does, so it is not the sugar pucker in itself that is prohibitive of triplex formation with fully modified LNA TFOs (36). The puckering amplitude of ENA $\left(\sim 45^{\circ}\right)$ is smaller than that of LNA $\left(\sim 60^{\circ}\right)$. This change in puckering amplitude entails two differences between LNA and ENA: (i) the $\delta$ backbone angle changes $\sim 11^{\circ}$ and (ii) the $C 3^{\prime}$ atom is less protruding in ENA. Both of these factors have been promulgated as possible reasons for LNA's triplex-forming properties $(35,37)$. The additional carbon atom in the ENA linker allows the sugar of this nucleotide more freedom than the LNA sugar, which is rigidly locked in the $\mathrm{C} 3^{\prime}$-endo conformation. This slight increase in sugar flexibility might just be what is needed to accommodate a fully modified TFO. A structural investigation of an ENA-modified triplex is awaited to shed light on the interesting triplex-forming properties of this nucleic acid analogue.

Introduction of LNA nucleotides in TFOs gives rise to a substantial increase in triplex thermostability (12). Specifically in this paper, we show that our intramolecular dsDNA:LNA triplex is stable at $\mathrm{pH}$ 8.0. Torigoe et al. have performed thermodynamic analysis of LNA triplexes and found that the increased stability relative to DNA triplexes is entropic in origin, whereas the introduction of LNA 
nucleotides in the TFO increases the enthalpy relative to DNA TFOs (11). The major contribution to the enthalpy of a nucleic acid is the hydrogen bonding and base stacking, and it is conceivable that the spiral-like hydrogen bond pattern of the dsDNA:LNA triplex decreases the strength of the Watson-Crick and Hoogsteen hydrogen bonds by the large propeller twist between nucleobases. The entropic difference between DNA and LNA TFOs cannot be gauged from our structure, as this requires knowledge of both the singlestranded species and the solvation of different species.

The protonation of the cytidines in the TFO strand contributes to the stability of a triplex. We observe that the cytidines of the LNA TFO remain protonated at $\mathrm{pH} 8.0$, and thus the $\mathrm{pH}$ range compatible with triplex formation is extended beyond neutral $\mathrm{pH}$. For synthetic reasons, the modified LNA cytidines are methylated at the 5-position in the nucleobase. Use of 5-methylcytidines instead of cytidines increases the stability of triplexes (and duplexes). For example, for an 11 mer TFO with five cytidines, the melting temperature was increased by $10^{\circ} \mathrm{C}$ and the $\mathrm{pH}$ value of semiprotonation raised from 5.8 to 6.8 (26). For the dsDNA:LNA triplex, the methylation of the three cytidines can by no means account for the elevation in melting temperature $\left(\sim 6-7^{\circ} \mathrm{C}\right.$ per LNA cytidine) and the increased $\mathrm{pH}$ stability. Thus, the effect seen is a combination of both the additional methyl group of the cytidines and the LNA modifications.

Deprotonation of the TFO cytidines occurs in two steps; local opening of the base pair followed by proton abstraction from the open state (27). Thus the apparent $\mathrm{p} K_{\mathrm{a}}$ of the cytidines depends on both the $\mathrm{p} K_{\mathrm{a}}$ of the non-base-paired cytidine and the base-pair dissociation constant. As we expect, the $\mathrm{p} K_{\mathrm{a}}$ of the non-paired LNA-modified 5-methyl-cytidine to be similar to that of 'unmodified' 5-methyl-cytidine, the high apparent $\mathrm{p} K_{\mathrm{a}}$ of the cytidines in the LNA TFO must be owing to lowered base-pair dissociation rates. The spiral-like hydrogen bond pattern in the dsDNA:LNA triplex could reduce base-pair opening (lower the base-pair dissociation rates) and consequently raise the apparent $\mathrm{p} K_{\mathrm{a}}$ of cytidine residues by making deprotonation events occurring less frequently.

Kinetic studies performed by Torigoe et al. shows that the dissociation rates of LNA TFOs were significantly lowered relative to the rates for DNA TFOs (11). This concerns the dissociation of the entire TFO strand and as such give no information on the kinetics of base-pair opening for single triplets. It is, however, possible that a lower propensity of base-pair opening at the triplet level would translate into a lower dissociation rate at the strand level.

When LNA TFOs are targeted at mixed A/G sequences, it appears that there is little difference in thermostability with respect to modifying thymidines or cytidines in the TFO (12). For sequences with contiguous stretches of $\mathrm{A}$ or $\mathrm{G}$ nucleotides, the highest stabilization of triplex formation is achieved if the LNA modifications are placed on TFO thymidines (37). This indicates that the stacking and hydrogen bonding motif we observe, could be specific for LNA-modified triplexes with mixed sequences.

\section{CONCLUSION}

In summary, we have determined the NMR solution structure of an intramolecular dsDNA:LNA triplex. The Watson-Crick part of the triplex adopts a geometry intermediate between A- and B-type. As compared with the corresponding dsDNA:DNA and dsDNA:RNA versions of this triplex, the dsDNA part of the triplex changes its geometry to facilitate efficient triplex formation with the LNA TFO. As a consequence of this geometry adaptation and the conformation of the TFO, the pyrimidines of the duplex part and the TFO are close in space and weak hydrogen bonding is taking place between the pyrimidines of the TFO and the $5^{\prime}$-flanking pyrimidines of the Watson-Crick duplex. This results in a spirallike hydrogen bonding pattern through the triplex. This kind of stacking and hydrogen bond pattern has not been observed in any triplex before, and as such is a motif peculiar to dsDNA:LNA triplexes.

Furthermore, we have shown, by use of NMR spectroscopy, that the dsDNA:LNA triplex is stable at $\mathrm{pH} 8$ and that the triplex structure is identical to the structure determined at pH 5.1.

The structure presented should prove useful in the design of optimum LNA TFOs and modelling of LNA triplex structures, e.g. in design of artificial nucleobases that recognize pyrimidines.

\section{COORDINATES}

Coordinates and restraints employed in calculations have been deposited in the Protein Data Bank (accession code: 1w86).

\section{SUPPLEMENTARY MATERIAL}

Supplementary Material is available at NAR Online.

\section{ACKNOWLEDGEMENTS}

Britta M. Dahl (Department of Chemistry, University of Copenhagen) is thanked for oligonucleotide synthesis. We thank The Instrument Centre for NMR Spectroscopy of Biological Macromolecules at The Carlsberg Laboratory, Copenhagen granted by The Danish Natural Science Research Council for providing spectrometer time at the $800 \mathrm{MHz}$ spectrometer. The Danish National Research Foundation is thanked for financial support.

\section{REFERENCES}

1. Praseuth,D., Guieysse,A.L. and Helene,C. (1999) Triple helix formation and the antigene strategy for sequence-specific control of gene expression. Biochim. Biophys. Acta, 1489, 181-206.

2. Giovannangeli,C. and Helene,C. (2000) Triplex-forming molecules for modulation of DNA information processing. Curr. Opin. Mol. Ther., 2, 288-296.

3. Seidman,M.M. and Glazer,P.M. (2003) The potential for gene repair via triple helix formation. J. Clin. Invest., 112, 487-494.

4. Buchini,S. and Leumann,C.J. (2003) Recent improvements in antigene technology. Curr. Opin. Chem. Biol., 7, 717-726.

5. Wang,E. and Feigon,J. (1999) Oxford Handbook of Nucleic Acid Structures, Neidle,S. (ed.), Oxford University Press, Oxford, pp. $355-388$.

6. Loakes,D. (2001) The applications of universal DNA base analogues. Nucleic Acids Res., 29, 2437-2447.

7. Wengel,J. (1999) Synthesis of $3^{\prime}-C$ - and $4^{\prime}-C$-branched oligonucleotides and the development of locked nucleic acid (LNA). Acc. Chem. Res., 32, 301-310. 
8. Obika,S., Nanbu,D., Hari,Y., Morio,K., In,Y., Ishida,T. and Imanishi,T. (1997) Synthesis of $2^{\prime}-O, 4^{\prime}-C$-methyleneuridine and -cytidine. Novel bicyclic nucleosides having a fixed $\mathrm{C}^{\prime}$-endo sugar puckering. Tetrahedron Lett., 38, 8735-8739.

9. Petersen,M. and Wengel,J. (2003) LNA: a versatile tool for therapeutics and genomics. Trends Biotechnol., 21, 74-81.

10. Jepsen,J.S. and Wengel,J. (2004) LNA-antisense rivals siRNA for gene silencing. Curr. Opin. Drug Discovery Dev., 7, 188-194.

11. Torigoe,H., Hari,Y., Sekiguchi,M., Obika,S. and Imanishi,T. (2001) $2^{\prime}-O, 4^{\prime}-C$-methylene bridged nucleic acid modification promotes pyrimidine motif triplex DNA formation at physiological $\mathrm{pH}$. J. Biol. Chem., 276, 2354-2360.

12. Obika,S., Uneda,T., Sugimoto,T., Nanbu,D., Minami,T., Doi,T. and Imanishi,T. (2001) $2^{\prime}-O, 4^{\prime}-C$-methylene bridged nucleic acid $\left(2^{\prime}, 4^{\prime}\right.$-BNA): synthesis and triplex-forming properties. Biorg. Med. Chem., 9, 1001-1011.

13. Koshkin,A.A., Singh,S.K., Nielsen,P., Rajwanshi,V.K., Kumar,R., Meldgaard,M., Olsen,C.E. and Wengel,J. (1998) LNA (locked nucleic acids): synthesis of the adenine, cytosine, guanine, 5-methylcytosine, thymine and uracil bicyclonucleoside monomers, oligomerization, and unprecedented nucleic acid recognition. Tetrahedron, 54, 3607-3630.

14. Geen,H. and Freeman,R. (1991) Band-selective radiofrequency pulses J. Magn. Reson., 93, 93-141.

15. Güntert,P. and Wüthrich,K. (1992) FLATT—a new procedure for high-quality baseline correction of multidimensional NMR spectra. J. Magn. Reson., 96, 403-407.

16. Wijmenga,S.S. and van Buuren,B.N.M. (1998) The use of NMR methods for conformational studies of nucleic acids. Prog. Nucl. Magn. Reson. Spectrosc., 32, 287-387.

17. Saenger,W. (1984) Principles of Nucleic Acid Structure. Springer-Verlag, New York

18. Case,D.A., Pearlman,D.A., Caldwell,J.W., Cheatham,T.E.,III, Ross,W.S., Simmerling,C.L., Darden,T.A., Merz,K.M., Stanton,R.V., Cheng,A.L. et al. (1997) AMBER 5. University of California, San Francisco.

19. Bayly,C.I., Cieplak,P., Cornell,W.D. and Kollman,P.A. (1993) A well-behaved electrostatic potential based method using charge restraints for deriving atomic charges: the RESP model. J. Phys. Chem., 97, 10269-10280.

20. Lavery,R. and Sklenar,H. (1988) The definition of generalized helicoidal parameters and of axis curvature of irregular nucleic acids. J. Biomol. Struct. Dyn., 6, 63-91.

21. Lavery,R. and Sklenar,H. (1989) Defining the structure of irregular nucleic acids: conventions and principles. J. Biomol. Struc. Dyn., 7, 655-667.

22. Wijmenga,S.S., Kruithof,M. and Hilbers,C.W. (1997) Analysis of ${ }^{1} \mathrm{H}$ chemical shifts in DNA: assessment of reliability of ${ }^{1} \mathrm{H}$ chemical shift calculations for use in structure refinement. J. Biomol. NMR, 10,337-350.

23. Cromsigt,J.A.M.T.C., Hilbers,C.W. and Wijmenga,S.S. (2001) Prediction of proton chemical shifts in RNA. Their use in structure refinement and validation. J. Biomol. NMR, 21, 11-29.
24. Tarköy,M., Phipps,A.K., Schultze,P. and Feigon,J. (1998) Solution structure of an intramolecular DNA triplex linked by hexakis (ethyleneglycol) units: d(AGAGAGAA-(EG)6-TTCTCTCT-(EG) 6- $^{-}$ TCTCTCTT). Biochemistry, 37, 5810-5819.

25. Gotfredsen,C.H., Schultze,P. and Feigon,J. (1998) Solution structure of an intramolecular pyrimidine-purine-pyrimidine triplex containing an RNA third strand. J. Am. Chem. Soc., 120, 4281-4289.

26. Xodo,L.E., Manzani,G., Quadrifoglio,F., van der Marel,G.A. and van Boom,J.H. (1991) Effect of 5-methylcytosine on the stability of triple-stranded DNA - a thermodynamic study. Nucleic Acids Res., 19, $5625-5631$.

27. Leitner,D., Schröder,W. and Weisz,K. (2000) Influence of sequence-dependent cytosine protonation and methylation on DNA triplex stability. Biochemistry, 39, 5886-5892.

28. Asensio,J.L., Brown,T. and Lane,A.N. (1999) Solution conformation of a parallel DNA triple helix with $5^{\prime}$ and $3^{\prime}$ triplex-duplex junctions. Structure, 7, 1-11.

29. MacDonald,D., Herbert,K., Zhang,X., Polgruto,T. and Lu,P. (2001) Solution structure of an A-tract DNA bend.J.Mol.Biol.,306, 1081-1098.

30. Rumney,S. and Kool,E.T. (1995) Structural optimization of non-nucleotide loop replacements for duplex and triplex DNAs. J. Am. Chem. Soc., 117, 5635-5646.

31. Bartley,J.P., Brown,T. and Lane,A.N. (1997) Solution conformation of an intramolecular DNA triplex containing a nonnucleotide linker: comparison with the DNA duplex. Biochemistry, 36, 14502-14511.

32. Plum,G.E. and Breslauer,K.J. (1995) Thermodynamics of an intramolecular DNA triple helix: calorimetric and spectroscopic study of the $\mathrm{pH}$ and salt dependence of thermally induced structural transitions. J. Mol. Biol., 248, 679-695.

33. Asensio,J.L., Lane,A.N., Dhesi,J., Bergqvist,S. and Brown,T. (1998) The contribution of cytosine protonation to the stability of parallel DNA triple helices. J. Mol. Biol., 275, 811-822.

34. Jensen,G.A., Singh,S.K., Kumar,R., Wengel,J. and Jacobsen,J.P. (2001) A comparison of the solution structures of an LNA:DNA duplex and the unmodified DNA:DNA duplex. J. Chem. Soc. Perkin Trans., 2, 1224-1232.

35. Koizumi,M., Morita,K., Daigo,M., Tsutsumi,S., Abe,K., Obika,S. and Imanishi,T. (2003) Triplex formation with $2^{\prime}-O$, 4'-C-ethylene-bridged nucleic acids (ENA) having $\mathrm{C}^{\prime}$ '-endo conformation at physiological pH. Nucleic Acids Res., 31, 3267-3272.

36. Morita,K., Takagi,M., Hasegawa,C., Kaneko,M., Tsutsumi,S., Sone,J., Ishikawa,T., Imanishi,T. and Koizumi,M. (2003) Synthesis and properties of $2^{\prime}-O, 4^{\prime}-C$-ethylene-bridged nucleic acids (ENA) as effective antisense oligonucleotides. Bioorg. Med. Chem., 11, 2211-2226.

37. Sun,B.-W., Babu,B.R., Sørensen,M.D., Zakrzewska,K., Wengel,J. and Sun,J.-S. (2004) Sequence and pH effects of LNA-containing triple helix-forming oligonucleotides: physical chemistry, biochemistry, and modeling studies. Biochemistry, 43, 4160-4169. 